\title{
INTELIGENCIA EMOCIONAL. EFECTOS SOBRE EL RENDIMIENTO ACADÉMICO EN ESTUDIANTES DE EDUCACIÓN
}

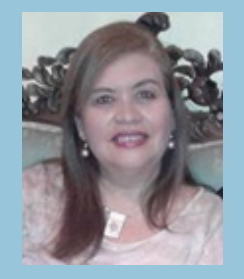

\section{Marhilde Sánchez}

Universidad del Zulia, Venezuela

marsanchezg@gmail.com

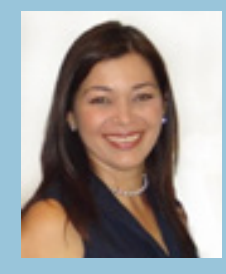

\section{Marisela Árraga}

Universidad del Zulia, Venezuela

marsanchezg@gmail.com

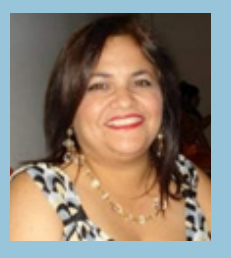

\section{Ligia Pirela}

Universidad del Zulia, Venezuela

marsanchezg@gmail.com

\section{RESUMEN}

Esta investigación indagó los efectos de un programa de intervención psicológica basado en la inteligencia emocional en el rendimiento académico de estudiantes de la Escuela de Educación, mención química, al efectuar comparaciones antes y después de la administración de dicho programa. Se recopiló información del rendimiento académico proveniente de las calificaciones o notas en un evaluativo escrito realizado por los estudiantes, en la asignatura Introducción a la Psicología y desarrollo humano. Se diseñó un programa referido a la inteligencia emocional, bajo la perspectiva de los creadores del término Salovey y Mayer (1990), el cual se administró durante 
16 horas. Posteriormente se efectuó otro evaluativo escrito y se registraron las calificaciones de los alumnos. Se utilizó un enfoque cuantitativo, nivel explicativo, método de campo, diseño experimental. La población estuvo integrada por 23 estudiantes, de los cuales 8 no hicieron acto de presencia en las clases, por lo cual se trabajó con 15 cursantes de la asignatura común para los estudiantes de las diversas menciones de la carrera Educación. Diez de sexo femenino y cinco de sexo masculino, con edades comprendidas entre 16 a 22 años, integrantes de la única sección de la asignatura en los tres turnos, de la mención química. Se aplicaron estadísticas descriptivas para los puntajes obtenidos antes y después de aplicar el programa y la Prueba T de Student para determinar si hubo diferencias. Se evidenció que el promedio para el grupo antes de aplicar el programa fue de 16,20, la mediana 17, con desviación estándar de 4,46, y luego de aplicarlo el promedio fue 18,92, la mediana 19, con desviación de 1,38, valor $\mathrm{t}$ de $-2,11$ y probabilidad 0,045 , menor que el nivel de significación de 0,05 , indicando que los puntajes se incrementaron significativamente después de la aplicación del programa.

Palabras clave: Inteligencia emocional, rendimiento académico, intervención psicológica.

\title{
EMOTIONAL INTELLIGENCE. THE EFFECTS OF A PSYCHOLOGICAL INTERVENTION PROGRAM ON THE ACADEMIC ACHIEVEMENT OF STUDENTS OF THE SCHOOL OF EDUCATION.
}

\begin{abstract}
This investigation studied the effects of a psychological intervention program based on emotional intelligence on the academic achievement of students of the School of Education, minoring in chemistry, when making comparisons before and after the administration of said program. Information was collected on the academic performance of the grades in a written evaluation carried out by the students, in the subject Introduction to Psychology and Human Development. A program related to emotional intelligence was designed, from the perspective of the creators of the term, Salovey and Mayer (1990), which lasted 16 hours. Subsequently another written evaluation was carried out and the students' grades were recorded. We used a quantitative approach, explanatory level, field method, with an experimental design. The population was composed of 23 students, of whom 8 did not make an appearance in class, so we worked with 15 students of the common subject for students of the various mentions of the career Education. 10 female and 5 male, aged between 16 and 22, members of the only section of the subject in the three
\end{abstract}


shifts, of the chemical minor. Descriptive statistics were applied for the scores obtained before and after applying the program and Student's t-test to determine whether there were any significant differences. It was evidenced that the average for the group before applying the program was 16.20 , the median 17 , with a standard deviation of 4.46 , and after applying it the average was 18.92 , the median 19 , with a deviation of 1,38 , t-value of -2.11 and probability 0.045 , lower than the significance level of 0.05 , indicating that the scores increased significantly after the application of the program.

Key words: Emotional intelligence, academic performance, psychological intervention.

\section{INTRODUCCIÓN}

Es importante destacar que el rendimiento académico es una variable de gran complejidad, tomando en cuenta los múltiples elementos que intervienen en el mismo. Holahan (2011) señala condiciones físicas del salón de clases que afectan el desempeño académico y expone investigaciones en las cuales se ha evidenciado factores tales como el nivel de iluminación, la calidad de los aislantes del ruido y la disposición de los espacios diseñados, influyendo de manera sustancial en la efectividad del aprendizaje. También, elementos familiares, económicos y políticos, afectan de manera significativa, y en este momento histórico, la colectividad venezolana se encuentra sumergida en una grave crisis, del cual no escapan los nuevos estudiantes universitarios.

Además, desde la psicología se han estudiado elementos internos, entre los que se destacan el bienestar, el estrés, la motivación, tipos de inteligencia, la inteligencia emocional, el locus de control y su incidencia en el rendimiento de los estudiantes. En anteriores investigaciones, se ha indagado esta vinculación en estudiantes de la escuela de Educación, de la Facultad de Humanidades y Educación de La universidad del Zulia.

Inicialmente, en el intento de explorar las variables relacionadas con el rendimiento académico se analizaron los estudiantes de educación y se determinaron los coeficientes de correlación rho de Spearman entre las variables inteligencia, medida a través de las pruebas no verbales Otis y Purdue, y en torno a las motivaciones sociales. Se encontró una relación baja significativa entre la inteligencia medida a través de la prueba de Otis y media entre la prueba Purdue y el rendimiento académico. Asimismo se encontró una relación significativa, pero baja entre la motivación al logro y el rendimiento académico No se evidenció relación entre la motivación a la afiliación y el poder 
con el rendimiento académico. (Sánchez de Gallardo y Pirela de Faría, 2009).

También se realizó una investigación titulada variables personales y su relación con el rendimiento académico, en el cual se efectuaron estudios correlacionales entre las variables internalidad y felicidad con el rendimiento académico. Los resultados referidos a la felicidad y el rendimiento académico reportaron una correlación positiva moderada entre ambas variables (Arraga, Sánchez de Gallardo y Pirela de Faría, 2012), mientras que internalidad y rendimiento académico, evidenciaron un locus de control no interno y una correlación baja positiva entre internalidad total y rendimiento académico (Sánchez de Gallardo, Pirela de Faría y Árraga Barrios, 2012a).

Asimismo, se efectuó un estudio que tuvo como propósito identificar los efectos de un programa de intervención psicológica para los docentes, en la conducta de sus alumnos, estudiantes del nivel primaria, subsistema básico. En el mismo, se contrastaron 2 grupos (experimental y control) antes y después de la administración de un taller de 12 horas sobre los tópicos motivación al logro, autoeficacia y comunicación a un grupo de docentes. Se evidenció en sus alumnos, la disminución de conductas desfavorables en un $43 \%$ de estos comportamientos, no obstante se reveló un escaso incremento de las conductas favorables (Sánchez de Gallardo, Pirela de Faría y Árraga, 2012b) Se propuso optimizar el proceso de capacitación en los docentes y trabajar con otros actores del proceso educativo, lo cual se pretende hacer en esta investigación.

Las investigadoras Sánchez de Gallardo, Árraga y Pirela de Faría (2014a), han efectuado otros estudios con el propósito de analizar los efectos de diferentes variables psicológicas en el rendimiento académico. Se examinaron los efectos de una propuesta de intervención psicológica para incrementar el rendimiento académico y la motivación al logro. Se realizó la medición inicial de los grupos experimental y control, en el cual resultaron equivalentes así como la propuesta basada en la comunicación, internalidad - externalidad y autoeficacia.

Las evidencias concluyeron que la intervención psicológica incrementó de manera significativa el rendimiento académico en el grupo experimental, al comparar antes y después ese grupo y al contrastar grupo experimental y grupo control (Sánchez, Pirela y Árraga, 2014b). En cuanto a la incidencia del programa de intervención en la motivación al logro, solo se encontraron aumentos significativos las dimensiones metas e instrumentación. (Sánchez de Gallardo, Pirela de Faría y Árraga, 2015) 
Aunado a esto, se llevó a cabo, una indagación de la prosecución y rendimiento académico de los estudiantes de la mención orientación, específicamente alumnos de asignaturas dictadas por una misma profesora: Cuantos se iniciaban en la asignatura del primer semestre, obligatoria para todas las menciones de la carrera educación, Introducción a la Psicología y desarrollo humano, luego continuaban en otras asignaturas específicas de la mención Enfoque sistémico de la realidad y posteriormente en la asignatura Teorías de la personalidad, correspondientes al primer, segundo y tercer semestre. En dicho estudio se identifica la crítica situación de los estudiantes de la mención. Este resultado fue informado a la dirección de escuela de educación y al decanato de humanidades y educación. (Sánchez de Gallardo, Árraga y Pirela de Faría, 2014c)

Resulta significativo enfatizar la actual situación de la escuela de educación, de la facultad de humanidades y educación, donde hay una mayor cantidad de alumnos en los semestres superiores próximos a graduarse, que en los primeros semestres de las carreras. Situación contraria a lo que ocurría anteriormente, cuando había un elevado número de alumnos en los primeros semestres, fundamentalmente en aquellas asignaturas comunes a las 10 menciones de la carrera Educación, que ameritaban varias secciones, e iban disminuyendo el número de alumnos en los semestres superiores.

Complementariamente, cabe destacar que el inicio de la carrera universitaria, está marcada por importantes cambios, tales como nuevos compañeros de estudios, mayor autonomía, espacios poco conocidos salones, bibliotecas, comedores y en el caso de nuestro país, lamentablemente acompañado por elementos como una hiperinflación galopante, que hace que muchos de los alumnos vayan a sus clases sin haber ingerido alimentos. y escaso suministro de agua para satisfacer necesidades mínimas de aseo y limpieza.

Concibiendo al estudiante como una totalidad y reconociendo cómo los elementos antes citados pueden incidir en el desenvolvimiento estudiantil, se trabajó con una variable interna la Inteligencia emocional, planteándose la inquietud: ¿una intervención psicológica basada en la inteligencia emocional incrementará el rendimiento académico? Del cual se generó como objetivo general de esta investigación: Analizar el efecto de un programa de intervención psicológica basado en la inteligencia emocional en el rendimiento académico de estudiantes de la Escuela de Educación, mención química.

Se planteó efectuar la intervención enfocada en dicha variable, destacándose los planteamientos 
de Extremera y Fernández Berrocal ( 2003) en cuanto a la importancia de afrontar muchas y variadas situaciones de riesgo, fuera de las aulas, tales como la pobreza extrema y entornos sociales deprimidos. Erazo (2012) enuncia problemas de tipo social, como pandillas, delincuencia, Peralta (2009) declara el consumo temprano de alcohol y sustancias psicoactivas (Citado por Erazo, 2012). Dichos autores proponen el aprendizaje de habilidades emocionales, sociales y prácticas para asegurar el desarrollo saludable durante la adolescencia y durante el curso de la vida.

Palomera , Fernández Berrocal y Brackett (2008) también destacan la relevancia de incluir la Inteligencia emocional, como competencia básica en la formación inicial del profesorado y en la escolaridad obligatoria, en base a la vulnerabilidad de los estudiantes, con elevados tasas de pobreza, elevada frecuencia de fracaso escolar, bajo rendimiento y deserción.

Además, se reportan estudios donde se han evidenciado disminución de alumnos que abandonan sus estudios, incremento en el promedio de asistencia a clases (Weare y Gray, 2001, citado por Palomera, Fernández Berrocal y Brackett, 2008) indicadores indirectos de resultados académicos, cuando participan en programas de Inteligencia emocional.

Bisquerra (2005) expone que la inteligencia emocional se puede enseñar y ante la evidencia existente se está obligado a hacerlo. Extremera y Férnandez Berrocal (2003) enuncian que una auténtica formación requiere desarrollo intelectual, pero también el potencial emocional, afectivo y social, para satisfacer las necesidades del mundo cambiante. Plantean la posibilidad real de ensenar habilidades de inteligencia emocional en el aula, ensenar programas de inteligencia socioemocional que de manera explícita contengan y resalten habilidades emocionales del modelo de Salovey y Mayer, basado en la capacidad para percibir, comprender y regular las emociones.

\section{Fundamentación teórica}

La Inteligencia emocional es un término muy conocido en la actualidad, gracias al fenómeno editorial producida por David Goleman, psicólogo y periodista norteamericano. Sin embargo, el término fue creado por Mayer y Salovey, quienes desarrollaron este útil constructo y lo fundamentaron con múltiples estudios, de los cuales se han derivado conclusiones en cuanto a su vinculación con el rendimiento académico, el ajuste psicológico y las conductas disruptivos (Extremera y Fernández Berrocal, 2003). 
Tomando en cuenta el ya mencionado impacto que causó Daniel Goleman en 1995 con su obra Inteligencia emocional, la cual ha sido un best seller en diferentes países, se reflexiona sobre las necesidades emocionales no satisfechas y los aportes previos de otras teorías, tales como la terapia racional emotiva de Albert Ellis, las humanistas de Carl Rogers, Abraham Maslow, estudios sobre la neurociencia y del cerebro emocional, así como la teoría de las inteligencias múltiples de Gardner. (Bisquerra, 2005).

Se reconoce el valor de componentes no cognitivos, factores afectivos, emocionales, personales y sociales que predecían las habilidades de adaptación y éxito en la vida. Salovey y Mayer (1990) explican inicialmente la Inteligencia emocional como un tipo de inteligencia social que incluye la capacidad de controlar las emociones propias y las de los demás, discriminar entre ellos y usar dicha información para guiar el pensamiento y el comportamiento. Posteriormente Salovey y Mayer (1997) proponen la inteligencia como una actividad mental y la define como habilidad para percibir, valorar y expresar emociones con exactitud, la habilidad para generar y o acceder sentimientos que faciliten el pensamiento, la habilidad para comprender las emociones, y el conocimiento emocional y la habilidad para regular las emociones promoviendo un crecimiento emocional e intelectual. (Citado por Extremera y Fernández Berrocal, 2003).

En la actualidad, los autores Salovey y Mayer (2000), mantienen las habilidades incluidas en esta reformulación y siguen definiendo la inteligencia emocional como la capacidad para procesar la información emocional con exactitud y eficiencia, incluyéndose la capacidad para percibir, asimilar, comprender y regular las emociones. De acuerdo a la definición presentada, consiste en la habilidad para unificar razonamiento y emociones, utilizar nuestras emociones para facilitar un razonamiento más efectivo y pensar en forma más inteligente sobre la vida emocional (Citados por Berrocal y Extremera ,2010).

La inteligencia emocional está compuesta por un conjunto de habilidades emocionales que forman un continuo, las cuales abarcan desde aquellas de nivel más básico, que ejecutan funciones fisiológicas fundamentales tales como la percepción y atención a los propios estados fisiológicos expresivos a otros de mayor complejidad cognitiva que buscan el manejo personal e interpersonal (Mayer, Caruso y Salovey, 2008, citados por Berrocal y Extremera ,2010).

Las cuatro habilidades de complejidad ascendente planteadas por estos autores son la habilidad para percibir, valorar y expresar emociones con exactitud, la habilidad para acceder y o 
generar sentimientos que faciliten el pensamiento, la habilidad para comprender emociones y el conocimiento emocional y la habilidad para regular las emociones promoviendo un crecimiento emocional e intelectual (Mayer y Salovey, 1997, citado por Extremera y Fernández Berrocal (2003).

La percepción emocional se encontraría en la base de la pirámide. Consiste en la habilidad para identificar y reconocer tanto los propios sentimientos como los de aquellos que te rodean. Incluye prestar atención y decodificar con precisión las señales emocionales de la expresión facial, movimientos corporales y tono de voz.

Dicha destreza emocional se refiere al grado en que los individuos pueden identificar sus propias emociones, así como los estados y sensaciones fisiológicas y cognitivas que estas conllevan. Esta habilidad implicaría la facultad para discriminar acertadamente la honestidad y sinceridad de las emociones expresadas por lo demás.

La facilitación o asimilación emocional implica la habilidad para tener en cuenta los sentimientos cuando se razona o solucionamos problemas. Esta habilidad se enfoca en cómo las emociones afectan el sistema cognitivo y cómo los estados afectivos ayudan a la toma de decisiones. También ayuda a priorizar los procesos cognitivos básicos, focalizando la atención en lo que es efectivamente importante.

Dependiendo de los estados emocionales, los puntos de vista de los problemas cambian, incluso mejorando el proceso creativo. Mas explícitamente, esta habilidad plantea que las emociones actúan de forma positiva sobre el razonamiento y la forma de procesar la información.

La comprensión emocional consiste en la habilidad para desglosar el amplio y complejo repertorio de señales emocionales, etiquetar las emociones y reconocer en qué categoría se agrupan los sentimientos. Implica una actividad tanto anticipatoria como retrospectiva para conocer las causas generadoras del estado anímico y las futuras consecuencias de las acciones. Asimismo, supone conocer cómo se combinan los diferentes estados emocionales dando lugar a emociones secundarias. Incluye la habilidad para interpretar el significado de las emociones complejas, la destreza para reconocer las transiciones de unos estados emocionales a otros y la aparición de sentimientos simultáneos y contradictorios. 
La regulación emocional es la habilidad más compleja de la Inteligencia emocional. Incluye la capacidad para estar abierto a los sentimientos, tanto positivos como negativos y reflexionar sobre los mismos, para descartar o aprovechar la información que los acompaña en función de su utilidad. Incluye la habilidad para regular emociones propias y ajenas, moderando las emociones negativas e intensificando las positivas. Abarca el manejo del mundo intrapersonal y también el interpersonal, esto es la capacidad para regular las emociones de los demás, poniendo en práctica diversas estrategias de regulación emocional que modifican tanto los propios sentimientos, como los de los demás.

Esta habilidad alcanzaría los procesos emocionales de mayor complejidad, es decir la regulación consciente de las emociones para lograr un crecimiento emocional e intelectual. (Mayer y Salovey, 1997, citado por Extremera y Fernández Berrocal, 2003).

Estas cuatro ramas forman una jerarquía. La regulación emocional es el componente de mayor nivel en la jerarquía y también el de mayor complejidad. De modo que la habilidad para regular las emociones individuales y las de los demás se construye sobre la base de las competencias representadas en las otras tres ramas. Además se observa una distinción entre la segunda rama y las otras 3. Mientras que la primera, tercera y cuarta rama (percepción, comprensión y regulación) incluye el proceso de razonar acerca de las emociones, la segunda rama incluye el uso de las emociones para mejorar el razonamiento.

Aunado a esto, se describe la inteligencia emocional como una habilidad para inducir en los otros las respuestas deseables que sirven de apoyo a varias aptitudes, tales como la comunicación, mayor popularidad, disposición al trabajo en equipo, mayor compromiso, mejor desenvolvimiento ante circunstancias adversas, entre otros. (Carrasquero, 2010)

Igualmente, se enuncia la inteligencia emocional como cualidades referidas a entender los propios sentimientos, tener empatía con los demás y regular las propias emociones, para mejorar la forma de vivir. Una persona con elevada inteligencia emocional podrá anticipar en comportamientos referidos a como evaluar a la gente, complacer a los demás e influir en ellos (DuBrin, 2008), elementos claves en el desempeño de los adolescentes y jóvenes estudiantes de educación.

Vinculado con lo anterior, en la actualidad no sólo se juzga a los individuos por su inteligencia, 
formación o experiencia, sino también por el modo en el cual se relacionan consigo mismo o con los demás. En consecuencia, se considera la inteligencia emocional como clave para el éxito personal y se cree que influye en el rendimiento académico y profesional. (Sánchez de Gallardo y Padrón, 2010).

Por último se exponen características de las persona que son emocionalmente inteligentes: actitud positiva, reconocer los propios sentimientos y emociones, capacidad para expresar sentimientos y emociones, capacidad de controlar sentimientos y emociones, empatía, capaz de tomar decisiones adecuadas, motivación, ilusión, interés; autoestima, saber dar y recibir, tener valores alternativos, ser capaz de superar dificultades y frustraciones y ser capaz de integrar polaridades. (Rovira, 1998, citado por Bisquerra, 2005).

Las personas con actitud positiva resaltan los aspectos positivos por encima de los negativos, valoran más los aciertos que los errores, más las cualidades que los defectos, más lo conseguido que las insuficiencias, más los pequeños éxitos que los fracasos, consideran más el esfuerzo que los resultados, hacen uso frecuente del elogio sincero, buscan el equilibrio entre la tolerancia y la exigencia. Todo ello siendo conscientes de las limitaciones individuales y las de los demás.

Reconocer los propios sentimientos y emociones: Lo cual en oportunidades no es fácil, hay emociones y sentimientos que cuesta reconocer. El análisis personal ayuda a este reconocimiento y en consecuencia, contribuye al desarrollo de la inteligencia emocional.

Capacidad para expresar sentimientos y emociones: Las emociones positivas y negativas requieren canalizarse a través de algún medio de expresión. El individuo emocionalmente inteligente sabe reconocer el canal más apropiado y el momento oportuno.

Capacidad para controlar sentimientos y emociones: El individuo emocionalmente inteligente puede encontrar el equilibrio entre la expresión de emociones y su control. En oportunidades hay que saber esperar. La tolerancia a la frustración y la capacidad de demorar la gratificación son indicadores de inteligencia emocional.

La empatía: Está referida a hacer nuestros los sentimientos y emociones de los demás. La persona empática capta las emociones de los otros que no son expresadas en palabras, sino mediante lenguaje corporal, en el tono de la voz, la mirada, la expresión de la cara. La empatía es 
un indicador de haber superado el egocentrismo y haber logrado una cierta madurez emocional.

Ser capaz de tomar decisiones adecuadas: Al momento de tomar decisiones están más presentes factores emocionales que racionales. Las decisiones pertinentes implican una integración entre lo racional y lo emocional. La emoción, en oportunidades, impide la decisión y de la indecisión surge el conflicto. Tomar conciencia de los factores emocionales en la toma de decisiones es lograr un mejor autoconocimiento de cara a unas decisiones más adecuadas.

Motivación, ilusión, interés: La emoción y la motivación están muy vinculadas. El deseo suscita la motivación. Suscitar ilusión o interés por algo o alguien es un factor de motivación que ayuda a sentirse bien. La pereza, el aburrimiento, la pasividad demuestran un nivel bajo madurez emocional. La persona emocionalmente inteligente es capaz de motivarse, ilusionarse, interesarse en las personas y en la realidad que lo circunda.

Autoestima Se refiere a tener sentimientos positivos hacia sí mismo y confianza en las propias capacidades para hacer frente a los retos que se plantean. La autoestima es una dimensión emocional, mientras que el auto concepto es una dimensión cognitiva.

Bisquerra (2005) hace énfasis en las aplicaciones de la inteligencia emocional, en minimizar los alumnos que abandonan el sistema educativo, desarrollar la resiliencia, en la prevención del consumo de drogas, las relaciones de pareja, en el trabajo, en la salud, en la prevención de los desórdenes en la comida, en el control de las emociones y el optimismo como habilidad.

\section{METODOLOGÍA}

La presente investigación es de tipo explicativo, de campo, diseño experimental.

El estudio se clasifica de tipo explicativo, definido según Hernández y otros (2010), como aquellos dirigidos a responder respecto a las causas de fenómenos físicos o sociales. Así mismo, Tamayo y Tamayo (2012) enuncian que se presenta mediante la manipulación de una variable experimental, bajo condiciones rigurosamente comprobadas, con el propósito de describir de qué modo o por qué causa se produce una situación o acontecimiento particular. En este caso, los cambios en el rendimiento de los estudiantes universitarios, luego de la intervención efectuada, específicamente el programa de inteligencia emocional. 
Respecto al rendimiento académico, concebido como producto, proviene de las calificaciones obtenidas en exámenes escritos en la asignatura Introducción a la Psicología y desarrollo humano. Se dictaron clases magistrales por parte de la docente junto a actividades prácticas en el salón de clases y otras enviadas para hacer en casa. Se efectuó el examen inicial. Posteriormente, cuando se culminó todo el programa de la asignatura, con diversas estrategias instruccionales, se seleccionaron dos temas, específicamente Adolescencia y Adultez temprana para el otro evaluativo escrito. Es importante destacar que se les recomendó a los alumnos utilizar el texto Psicología y Educación de Sánchez, Árraga y Maldonado (2010) compendio diseñado para esta asignatura. Las calificaciones oscilan entre 0 y 20 puntos.

También se califica de campo, según lo señalado por Munch y Ángeles (2007) tomando en cuenta que la recolección de datos se realizó directamente en los sujetos investigados o de la realidad donde ocurren los hechos y este estudio se efectuó en el lugar donde se desarrolló la situación, particularmente en el interior de un salón del Bloque P de la facultad de humanidades y educación, donde dichos alumnos asisten a sus clases presenciales y donde se administró el programa de intervención correspondiente.

La población estuvo integrada por 23 estudiantes, de los cuales 8 no hicieron acto de presencia en las clases, por lo cual se trabajó con 16 cursantes de la asignatura Psicología Educativa. Diez de sexo femenino y seis de sexo masculino, con edades comprendidas entre 16 a 22 años, integrantes de la única sección de la asignatura en los tres turnos, de la mención química de la carrera Educación de la facultad de humanidades y educación de La Universidad del Zulia.

El procedimiento a seguir fue el siguiente: Se les expuso a los estudiantes el propósito y utilidad del estudio, aclarándose la participación voluntaria en el mismo, sin influencia en la calificación final de la asignatura. Se efectuó la primera evaluación, posteriormente, la intervención de carácter teórico-práctico, durante 16 horas, durante el horario de clases. La misma fue realizada por Licenciados en Educación mención orientación, actuando como facilitador y cofacilitador y acompañado por la investigadora responsable del programa.

Finalizando el semestre, se administró la segunda evaluación. Cabe destacar que dichas evaluaciones eran corregidas utilizando un formato de respuestas correctas, para eliminar influencias subjetivas en la corrección de dichos exámenes. Se introdujeron las calificaciones de los estudiantes, en una base de datos con el propósito de llevar a cabo el tratamiento estadístico 
correspondiente y generar los resultados que permitan comprender los efectos de la intervención psicológica planificada para este proyecto, enfocada en la inteligencia emocional, en el rendimiento académico.

\section{Resultados para el programa de inteligencia emocional}

Para el análisis de los resultados se aplicaron estadísticas descriptivas para los puntajes obtenidos en el rendimiento académico, antes y después de aplicar el programa y la Prueba $\mathrm{T}$ de Student para determinar si hubo diferencias.

En la Tabla 1, se puede observar que el promedio para el grupo antes de aplicar el programa fue de 16,20, la mediana 17, con desviación estándar de 4,46, y luego de aplicarlo el promedio fue de 18,92, la mediana 19, con desviación de 1,38 , con valor $\mathrm{t}$ de $-2,11$ y probabilidad 0,045 que es menor que el nivel de significación de 0,05 que indica que los puntajes se incrementaron significativamente después de la aplicación del programa.

\section{Tabla 1 . Resultados del rendimiento académico antes y después de aplicar el programa de Inteligencia emocional}

\begin{tabular}{|c|c|c|c|c|c|c|c|c|}
\hline \multicolumn{9}{|c|}{ Estadísticos de grupo } \\
\hline Medición & $\mathrm{N}$ & Media & Mediana & $\begin{array}{c}\text { Desviación } \\
\text { típica }\end{array}$ & $\begin{array}{c}\text { Error } \\
\text { típ. de la } \\
\text { media }\end{array}$ & $\mathrm{t}$ & $\mathrm{gl}$ & Sig. \\
\hline Antes & 16 & 16,20 & 17 & 4,46 & 1,15 & $-2,11$ & 26 & 0,045 \\
\hline Después & 16 & 18,92 & 19 & 1,38 &, 38 & & & \\
\hline
\end{tabular}

Fuente: Las Autoras

\section{Discusión, interpretación y argumentación de los resultados.}

La Inteligencia emocional, desde la perspectiva de Salovey y Mayer se encuentra integrada por cuatro habilidades de complejidad ascendente: La habilidad para percibir, valorar y expresar emociones con exactitud, la habilidad para acceder y o generar sentimientos que faciliten el pensamiento, la habilidad para comprender emociones y el conocimiento emocional y la habilidad para regular las emociones promoviendo un crecimiento emocional e intelectual ( Mayer y Salovey, 1997, citados por Extremera y Fernández Berrocal, 2003). Dichas habilidades afectan los diferentes 
procesos de aprendizaje, las vinculaciones sociales y hasta la salud física y mental e influyen en el funcionamiento personal, académico y social de los alumnos.

En este estudio se indagó el efecto de un programa de Inteligencia emocional en el rendimiento de estudiantes de primer semestre, de la carrera Educación, mención química. Dicho programa se administró durante 16 horas, en las clases presenciales de la asignatura Introducción a la Psicología y desarrollo humano, de carácter obligatorio para las diferentes menciones de la Escuela.

Los resultados evidenciaron un incremento significativo en las calificaciones de los estudiantes, específicamente la media antes de la aplicación del programa de Inteligencia emocional fue de 16,20 y la mediana 17; mientras que luego de la intervención la media fue de 18,92 y la mediana de 19. Al comparar ambos grupos de calificaciones con la Prueba T de Student, se encontraron diferencias significativas.

Dicha investigación constituye un progreso, en cuanto a otros estudios realizados de tipo descriptivo y correlacional entre variables psicológicas, tales como tipos de inteligencia, motivaciones sociales, locus de control, bienestar subjetivo; tomando en cuenta su carácter explicativo, al aplicar un programa de intervención psicológica enfocado en la Inteligencia emocional para determinar su efecto en el rendimiento académico de los estudiantes de la mención química.

Cabe destacar la importancia de la Inteligencia emocional en el desarrollo integral en los individuos, por lo que debe continuar implementándose este tipo de estrategias en los alumnos de esta mención y de las otras menciones, a fin de minimizar las posibilidades de deserción, tomando en cuenta la vulnerabilidad y otras situaciones de riesgo en los estudiantes como la pobreza extrema, dificultad de cubrir necesidades básicas de alimentación, limpieza, la delincuencia que se vive en Venezuela. También para optimizar las potencialidades y prevenir situaciones de conflictos, depresiones, consumo excesivo de alcohol y otras drogas, resulta imperante continuar con entrenamientos en las habilidades de inteligencia emocional.

Además, se sugiere hacer un seguimiento a los alumnos que participaron, con la finalidad de indagar las habilidades y características de las personas con inteligencia emocional que influyen en su desarrollo individual e identificar su prosecución y calificaciones en las diferentes asignaturas, tanto las comunes a todas las menciones, como las específicas de la carrera. 


\section{BIBLIOGRAFÍA}

\section{Libros}

Bisquerra, R. (2005). Educación emocional y bienestar. España. PRAXIS

DuBrin, A. (2008). Relaciones humanas. Comportamiento humano en el trabajo. México Edit. Pearson. Prentice Hall

Hernandez,R, Fernandez,C. Baptista,P (2010) Metodología de la investigación. México. Editorial Mac Graw Hill, S.A.

Holahan, Ch (2011). Psicología ambiental. Un enfoque general. México. Limusa

Munch , L. y Ángeles, E. (2007). Métodos y técnicas de investigación. México. Editorial Trillas.

Tamayo y Tamayo, M (2012). El Proceso de la Investigación Científica. México. Editorial Limusa.

\section{Revistas periódicas}

Àrraga, M, Sánchez de Gallardo, M Pirela de Faría, L (2012). Felicidad y Rendimiento Académico en Estudiantes Universitarios. Revista Orientación y Comunidad. Año $1 \mathrm{~N}^{\mathrm{o}} 1$. (pp. 71-86).

Carrasquero, C. (2010). Aptitudes Emocionales que Inciden en el Aprendizaje Organizacional. CIGAG. Volumen 7. No 1. (pp. 15-29).

Erazo, O. (2012). El Rendimiento Académico. Un Fenómeno de Múltiples Relaciones y Complejidades. Revista Vanguardia Psicológica. Volumen 2. Número 2. (pp. 144-173).

Palomera, R, Fernández- Berrocal,P, Brackett, M (2008). Inteligencia Emocional Como Una Competencia Básica en la Formación Inicial de los Docentes: Algunas Evidencias. Revista Electrónica de Investigación Psicoeducativa. Volumen 15. Número 6. (pp. 437-454).

Sánchez de Gallardo, M. y Padrón, A. (2010). Efecto de la motivación al logro y la Inteligencia emocional en el crecimiento psicológico Revista Venezolana de Gerencia. Año 15. No 49. (pp. 141-157).

Sánchez de Gallardo, M y Pirela de F, Ligia (2009). Motivos Sociales y Rendimiento Académico en Estudiantes Universitarios. Caso: Universidad del Zulia, mención Orientación. Revista Investigación y Postgrado Universidad Pedagógica Experimental Libertador. Numero 24 (3).

Sánchez de Gallardo, Marhilde, Pirela de Faría, Marisela y Árraga, Marisela. (2012a). Locus de control. Variable Personal Asociada al Rendimiento Académico. Revista Académica. Vol. 


\section{4. $\mathrm{N}^{\mathrm{o}} 8$ (pp. 1-14)}

Sánchez de Gallardo, Marhilde, Pirela de Faría, Ligia, Árraga, Marisela (2012b) Rendimiento Académico. Efectos de Intervención Psicológica Dirigida a Docentes en las Calificaciones de los Estudiantes. Revista UNICA.Volumen 13 No 34. (pp. 125-146).

Sánchez de Gallardo, Marhilde, Pirela de Faría, Ligia y Arraga Barrios, Marisela (2014a) Propuesta de Intervención Psicológica para Incrementar el Rendimiento Académico y la Motivación al Logro. Revista REDHES. Volumen 18, Año No. 9. (pp. 121-142).

Sánchez de Gallardo, Marhilde, Árraga, Marisela y Pirela de Faría, Ligia. (2014c) Rendimiento y Prosecución en una Universidad Pública Venezolana. Revista Encuentro educacional. Volumen 21.

\section{Ponencias o conferencias presentadas}

Sánchez de Gallardo, M, Pirela L. Árraga, M (2014b) Impacto de Intervención Psicológica en la El Rendimiento Académico de Estudiantes Universitarios. I Congreso Internacional Dr. Adolfo Calimán. Universidad Dr. José Gregorio Hernández, Noviembre Maracaibo, Venezuela

Sánchez de Gallardo, M Pirela L. Árraga, M (2015) Efectos de intervención psicológica en la motivación al logro de estudiantes universitarios. X Jornadas de Investigación y postgrado. III Encuentro internacional de investigadores. Universidad del Zulia. Noviembre. Punto Fijo, Venezuela

\section{Internet}

Extremera, N. y Fernández Berrocal, P. (2003). La Inteligencia Emocional en el Contexto Educativo: Hallazgos Científicos De Sus Efectos En El Aula. Revista de Educación. Número 332. pp 97116 .Disponible en: http://www.mecd.gob.es/dctm/revista-de-educacion/ articulosre332/re3320611443.pdf?documentId=0901e72b81256ae3 Consulta: 10/01/17. Fernández Berrocal, P. y Extremera N. (2010). Más Aristóteles y Menos Prozac. La Inteligencia Emocional y el Estudio de la Felicidad. Disponible en www.researchgate. net/publication/230886918_La_Inteligencia_Emocional_y_el_estudio_de_la_felicidad .Consulta $(10 / 02 / 17)$. 